\title{
CALET on the International Space Station: a precise measurement of the iron spectrum
}

\author{
Caterina Checchia, ${ }^{a, *}$ Francesco Stolzi ${ }^{a, b}$ and Yosui Akaike ${ }^{c, d}$ on behalf of the \\ CALET Collaboration \\ (a complete list of authors can be found at the end of the proceedings) \\ ${ }^{a}$ Department of Physical Sciences, Earth and Environment, \\ University of Siena, via Roma 56, 53100 Siena, Italy \\ ${ }^{b}$ INFN Sezione di Pisa, \\ Polo Fibonacci, Largo B. Pontecorvo, 3, 56127 Pisa, Italy \\ ${ }^{c}$ Waseda Research Institute for Science and Engineering, \\ Waseda University, 17 Kikuicho, Shinjuku, Tokyo 162-0044, Japan \\ dJEM Utilization Center, Human Spaceflight Technology Directorat, Japan Aerospace Exploration Agency, \\ 2-1-1 Sengen, Tsukuba, Ibaraki 305-8505, Japan \\ E-mail: caterina.checchia2@unisi.it, francesco.stolzi@unisi.it, \\ yakaike@aoni.waseda.jp
}

The Calorimetric Electron Telescope (CALET) was launched on the International Space Station in 2015 and since then has collected a large sample of cosmic-ray charged particles over a wide energy. Thanks to a couple of layers of segmented plastic scintillators placed on top of the detector, the instrument is able to identify the charge of individual elements from proton to iron (and above). The imaging tungsten scintillating fiber calorimeter provides accurate particle tracking and the lead tungstate homogeneous calorimeter can measured the energy with a wide dynamic range. One of the CALET scientific objectives is to measure the energy spectra of cosmic rays to shed light on their acceleration and propagation in the Galaxy. By the observation in first five years, a precise measurement of the iron spectrum is now available in the range of kinetic energy per nucleon from $10 \mathrm{GeV} / n$ to $2 \mathrm{TeV} / n$. The CALET's result with a description of the analysis and details on systematic uncertainties will be illustrated. Also, a comparison with previous experiments' results is given.

\footnotetext{
*** The European Physical Society Conference on High Energy Physics (EPS-HEP2021), ***

*** 26-30 July $2021 * * *$

*** Online conference, jointly organized by Universität Hamburg and the research center DESY ***
}

\footnotetext{
${ }^{*}$ Speaker
} 


\section{Introduction}

The CALorimetric Electron Telescope is a space-based experiments installed on the International Space Station that started scientific observation from October 2015. The instrument is a 30 radiation length deep calorimeter optimized for the measurement of the all-electron spectrum [1,2], but also able to measure the flux of individual chemical elements in cosmic rays (CR) from proton to iron and above in the energy range from few $\mathrm{GeV}$ up to the $\mathrm{PeV}$ scale. Direct measurements of charged cosmic rays (CR) available so far, have revealed unexpected spectral hardening in the light elements such as proton, helium [3-9] and heavier nuclei [10-14] spectra around a few hundred $\mathrm{GeV} / n$. In this context a precision measurement of the iron spectrum is of particular interest because it could help discriminating among several classes of theoretical models proposed to explain these spectral structures [15-21]. In fact, iron provides favorable conditions for observations due to its largest relative abundance among the heavy elements and also for a negligible contamination from spallation of higher mass elements. In this paper, we describe the analysis procedure for iron flux measurement and present the energy spectra from $10 \mathrm{GeV} / n$ to 2.0 TeV/n, based on the data collected by CALET from January 1, 2016 to May 31, 2020 aboard the International Space Station (ISS).

\section{Detector}

CALET instrument is composed by a Charge Detector (CHD) for the measurement of the charge, an IMaging calorimeter (IMC) that acts as a pre-shower calorimeter and a Total AbSorption Calorimeter (TASC). The CHD is a hodoscope consisting of a couple of layers made of 14 plastic scintillator paddles each, arranged in orthogonal way (CHDX and CHDY). The identification of particle charge $Z$ is based on the measurements of ionization deposits: the particle's trajectory provides the paddles traversed by the incoming particle and determine the path length correction to be applied to the signal to extract the $d E / d x$ sample. An additional measurement of the charge is provided by the IMC via multiple samples of specific energy loss $(d E / d x)$ in each fiber up to the onset of saturation which occurs for ions more highly charged than silicon. The CHD charge resolution $\sigma_{Z}$ is $\sim 0.35 e$ for iron.

The IMC consists of 7 tungsten plates spaced out by couple of layers of $1 \mathrm{~mm}^{2}$ cross-section scintillating fibers, arranged in belts along orthogonal directions. Fiber signals are used to reconstruct the CR particle trajectory and its entrance point in the instrument, by a tracking algorithm based on a combinatorial Kalman filter [22]. The angular resolution is $\sim 0.1^{\circ}$ for iron nuclei and the spatial resolution on the determination of the impact point on CHD is $\sim 180 \mu \mathrm{m}$.

The TASC is a homogeneous calorimeter made of 12 layers of lead-tungstate bars for a total thickness of 30 radiation lengths and 1.3 nuclear interaction lengths. Thanks to the readout by means of photosensors and the front-end electronics, a wide dynamic range of $>10^{6}$ is spanned. The energy response of TASC was calibrated at CERN SPS in 2015 using a beam of accelerated ion fragments with $\mathrm{A} / \mathrm{Z}=2$ and kinetic energy of 13,19 and $150 \mathrm{GeV} / n$ [23]. A good linearity up to the maximum available energy $(6 \mathrm{TeV})$ was found with an energy deposit of $\sim 20 \%$ and an energy resolution of $\sim 30 \%$ [14]. A Monte Carlo (MC) simulations based on EPICS package [24, 25], employing the hadronic interaction model DPMJET-III [26], was used to reproduce the detector 
configuration, physics processes, as well as detector signals. Independently, a simulations based on FLUKA [27] was performed to assess the systematic uncertainty. The energy response derived from MC simulations was tuned using the beam test results.

\section{Data Analysis}

The present analysis includes flight data (FD) collected between January 2015 and May 2020 for a total of 1613 days and a total observation live time for high energy (HE) shower trigger of $4.4 \times 10^{4} h$ corresponding to $85.8 \%$ of total observation time. A dedicated in-flight trigger mode for penetrating protons and He particles is used in order to calibrate each channel of CHD, IMC and TASC. Raw signals are then corrected for non-uniformity in light output, gain differences among the channels, position and temperature dependence as well as temporal gain variations.

Events with one well-fitted track crossing the whole detector from CHD top to the TASC bottom layer and at least $2 \mathrm{~cm}$ away from the edges of top and bottom TASC layers are then selected. The fiducial geometrical factor for this category of events is $S \Omega \sim 416 \mathrm{~cm}^{2}$ sr corresponding to about $40 \%$ of CALET total acceptance.

The HE trigger used for this analysis is based on the coincidence of the summed signals of the last two IMC layers in each view and the top TASC layer (TASCX1). Since the HE trigger is fully efficient (close to $100 \%$ ) for elements heavier than oxygen an off-line trigger confirmation, as required for the analysis of lower charge elements [9, 14], is not necessary for iron. However, in order to select interacting particles, a deposit larger than 2 sigmas of the minimum ionization particle (MIP) peak is required in at least one of the first four layers of the TASC.

In order to reconstruct the charge, a correction for the quenching effect occurring to CHD paddles due to high ionization density around the particle track is necessary[14]. A fit of halo model to CALET flight data as a function of $Z^{2}$ is used as a curve to restore the charge to its nominal position in either layer of CHD. In addition, an energy dependent charge correction is applied separately to flight data and MC data in order to correct the systematic displacement of the CHDX and CHDY charge peaks to higher values with respect to their nominal position due to an increasing amount of backscatters from the TASC at high energy. Particles undergoing a charge-changing nuclear interaction in the upper part of the instrument are rejected by requiring that the difference between the charge reconstructed in CHDX and in CHDY is less than 1.5 charge units. Iron candidates are then selected applying an elliptical cut centered on $Z=26$ with $1.25 \sigma_{x}$ and $1.25 \sigma_{y}$ wide semiaxis for CHDX and CHDY respectively and rotated clockwise by $45^{\circ}$ as shown in the cross plot in Fig. 1. With these selection criteria a sample of $4 \times 10^{4}$ iron candidates is selected.

The shower energy $E_{T A S C}$ of each event is calculated as the sum of the calibrated energy deposits of all the TASC logs [28]. The simulated energy response was corrected following the calibration curve obtained from the beam test analysis: the correction factors are $6.7 \%$ for $E_{T A S C}<45 \mathrm{GeV}$ and $3.5 \%$ for $E_{T A S C}>350 \mathrm{GeV}$, respectively. A linear interpolation is applied to determine the correction factor for intermediate energies. For nuclei with $Z>10$, the TASC crystals undergo a light quenching phenomenon which is not reproduced by the MC simulations. Therefore, it is necessary to extract from the data a quenching correction to be applied a posteriori to the MC energy deposits generated in the TASC logs by noninteracting primary particles [29]. 


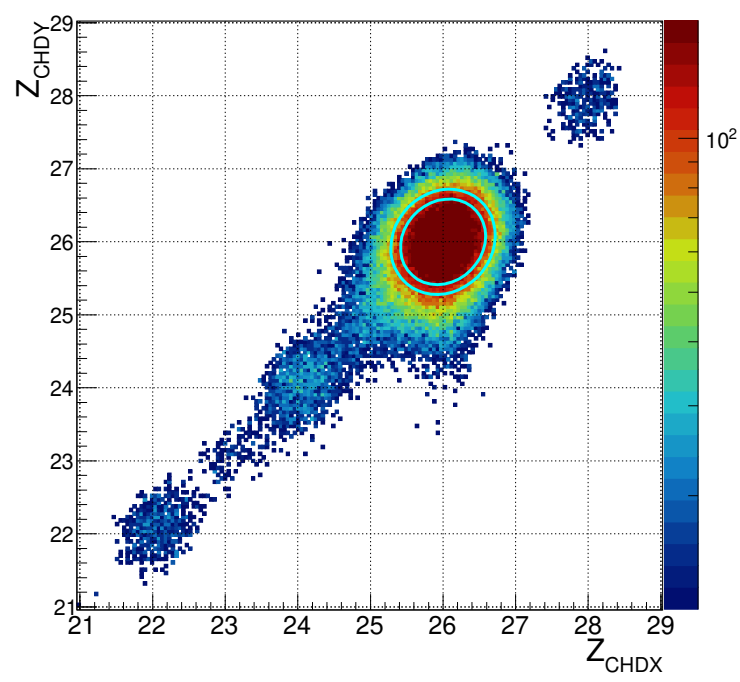

Figure 1: Cross plot of Z CHDY vs. Z CHDX reconstructed charges. The maximum and the minimum elliptical selection for the iron candidates are indicated by the cyan ellipses in the figure.

In order to reconstruct the primary particle energy starting from the energy released in TASC (that is only a fraction of it) and to take into account the bin-to-bin migration effect (due to limited energy resolution), energy unfolding based on the Bayes theorem [30] is applied to the $E_{T A S C}$ distributions. The energy spectrum is obtained from the unfolded energy distribution as follows:

$$
\begin{gathered}
\Phi(E)=\frac{N(E)}{\Delta E \varepsilon(E) S \Omega T} \\
N(E)=U\left[N_{o b s}\left(E_{\mathrm{TASC}}\right)-N_{b g}\left(E_{\mathrm{TASC}}\right)\right]
\end{gathered}
$$

where $\Delta E$ is the energy bin width; $E$ the particle kinetic energy, calculated as the geometric mean of the lower and upper bounds of the bin; $N(E)$ the bin content in the unfolded distribution; $T$ the exposure time; $\varepsilon(E)$ the total selection efficiency; $U()$ the iterative unfolding procedure; $N_{o b s}\left(E_{T A S C}\right)$ the bin content of observed energy distribution (including background); $N_{b g}\left(E_{T A S C}\right)$ the bin content of background events in the observed energy distribution. The total background from other nuclei mis-identified as iron for the selected sample is $<1 \%$ in the energy range from $10^{2} \mathrm{GeV}$ to $10^{3} \mathrm{GeV}$ of $E_{T A S C}$ increasing up to $\sim 2 \%$ at $E_{T A S C} \sim 10^{4} \mathrm{GeV}$.

\section{Systematic Errors}

The systematic uncertainties studied in this analysis include charge identification, MC model, energy scale correction, beam test configuration, unfolding procedure, and shower event identification. In order to estimate the systematic error related to the charge identification, the semi-axes of the elliptical cut were varied by $\pm 15 \%$ resulting in a flux variation lower by a few percent below $600 \mathrm{GeV} / n$ increasing to $\sim 10 \%$ at $1 \mathrm{TeV} / n$. Since it is not possible to validate MC simulations with beam test data in the high-energy region, a comparison between different MC models, i.e. EPICS and FLUKA, was performed. The total selection efficiencies of the two models are in agreement within $2 \%$ over the whole energy range but the energy response matrices differ significantly in the 
low and high energy regions. That produces a maximum discrepancy of $\sim 10 \%$ below $40 \mathrm{GeV} / n$ reducing to $5 \%$ at the $\mathrm{TeV} / n$ scale. The uncertainty in the energy scale is $\pm 2 \%$ and depends on the accuracy of the beam test calibration. It causes a rigid shift of the measured energies, affecting the absolute normalization of the Fe spectra by ${ }_{-3.2 \%}^{+3.3 \%}$, but not its shape. Also, the difference in the configuration model adopted at the test beam with respect to the instrument now in orbit, was modeled and included in the systematic error. The uncertainties due to the unfolding procedure were evaluated with different response matrices computed by varying the spectral index (between -2.9 and -2.2) of the MC generation spectrum, or by using the Singular Value Deconvolution method, instead of the Bayesian approach. At last, the systematic contribution due to non interacting particles, rejected through the shower event selection, was evaluated and included in the systematic uncertainties. Other energy-independent systematic uncertainties affecting the normalization include live time $(3.4 \%)$, long-term stability $(<2 \%)$ and geometrical factor $(\sim 1.6 \%)$. The contributions to the systematic uncertainties due to off-acceptance events, tracking efficiency, background contamination and HE trigger efficiency are negligible. The total systematic error is computed as the sum in quadrature of all the sources of systematics in each energy bin. A breakdown summary of systematic errors is shown in Fig 2.

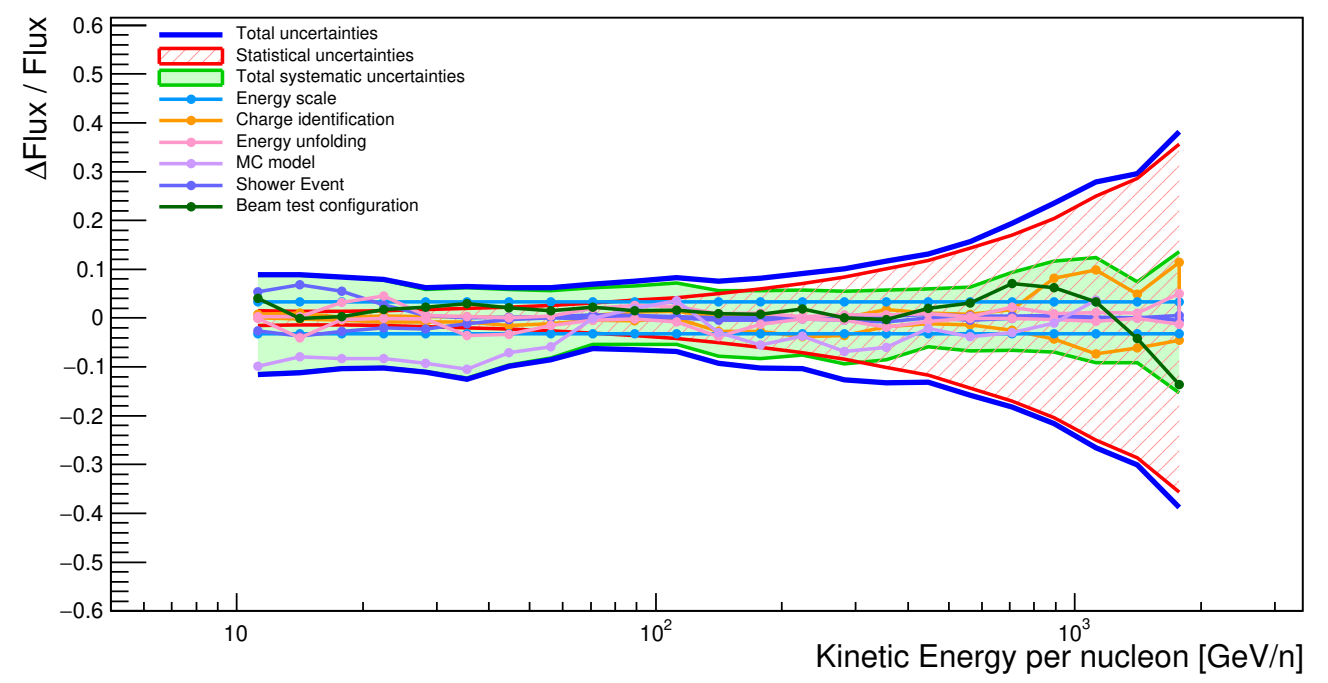

Figure 2: Energy dependence (in $\mathrm{GeV} / n$ ) of systematic uncertainties (relative errors) for iron. The band bounded by the red lines represents the statistical error. The shaded band within the green lines shows the sum in quadrature of all the sources of systematics including energy independent ones. The blue lines represent the sum in quadrature of statistical and total systematic uncertainties.

\section{Results}

The energy spectrum of iron measured with CALET in the energy range from $10 \mathrm{GeV} / n$ to $2.0 \mathrm{TeV} / n$ is shown in Fig. 3 where the green band stands for the sum in quadrature of statistical and systematic error (the latter represented by the yellow band). CALET is consistent with ATIC 02 [31] and TRACER [32] at low energy and with CRN [38] and HESS [36] at high energy, but differs in the absolute normalization with NUCLEON (lower) and Sanriku [35] (higher). CALET and AMS02 [39] iron spectra have a very similar shape and comparable errors, but differ in the absolute normalization of the flux by $\sim 20 \%$ as shown in Fig. 4 . 


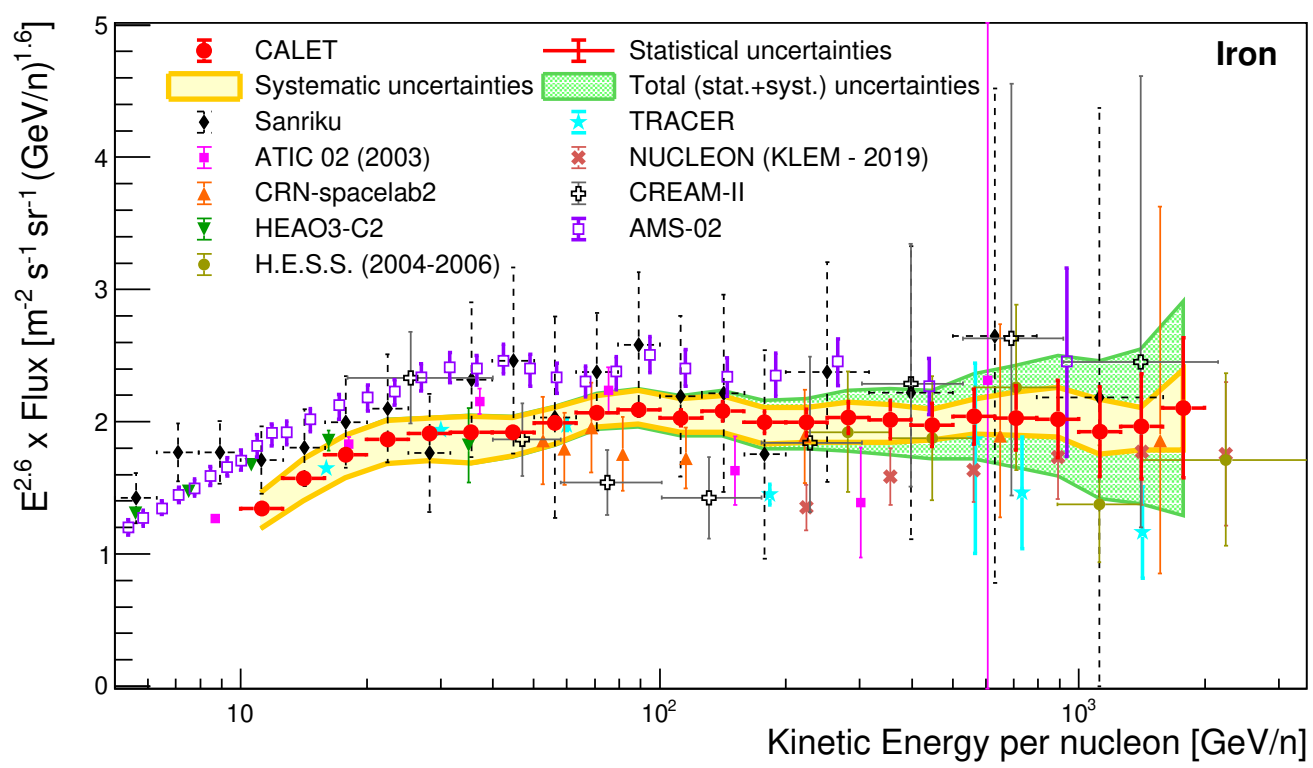

Figure 3: CALET iron flux (multiplied by $E^{2.6}$ ) as a function of kinetic energy per nucleon. Error bars of the CALET data (red) represent the statistical uncertainty only, the yellow band indicates the quadrature sum of systematic errors, while the green band indicates the quadrature sum of statistical and systematic errors. Also plotted are other direct measurements [31-39].

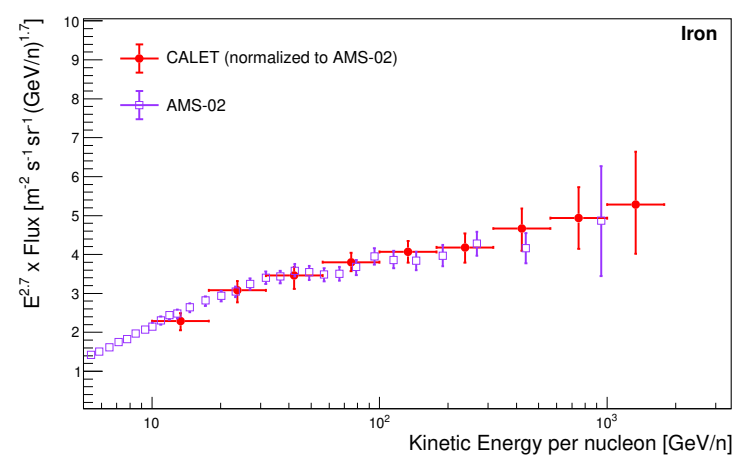

Figure 4: Iron flux (with multiplicative factor $E^{2.7}$ ) measured by CALET (red points) with 4 bins/decade, multiplied by 1.20 for comparison with the AMS-02 results [39]. The error bars of CALET data are the quadrature sum of statistical and systematic uncertainties.

The CALET spectrum has been fitted from $50 \mathrm{GeV} / n$ to $2 \mathrm{TeV} / n$ in order to investigate spectral features (Fig. 5). The fitted function is a single power law (SPL) and the resulting spectral index is $\gamma=-2.60 \pm 0.02$ (stat) \pm 0.02 (sys) with $\chi^{2} / D O F=4.2 / 14$. The result is stable when the binning is changed from 10 to 4 bins/decade $(\gamma=-2.59 \pm 0.02$ (stat) $\pm 0.04($ sys $)$ ). In order to understand whether the flux may suggest any change in spectral behavior in the region between $50 \mathrm{GeV} / n$ and $2 \mathrm{TeV} / n$, the spectral index $\gamma$ is calculated by a fit of $d[\log (\Phi)] / d[\log (E)]$ inside a sliding window centered in each energy bin and including the neighboring \pm 3 bins (Fig. 6).

\section{Conclusion}

CALET measures the iron spectrum with significantly better precision than most of the existing measurements. The spectrum is consistent with the hypothesis of a single power law with spectral 


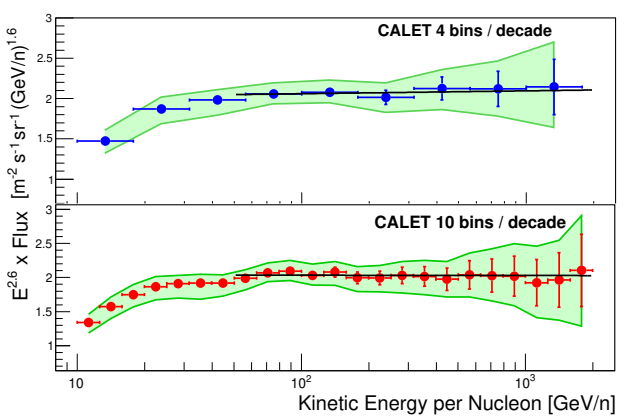

Figure 5: Fit of the CALET iron energy spectrum to a SPL function (black lines) in the energy range $[50,2000] \mathrm{GeV} / n$ with 4 bins/decade (top) and 10 bins/decade (bottom).The green band indicates the quadrature sum of statistical and systematic errors.

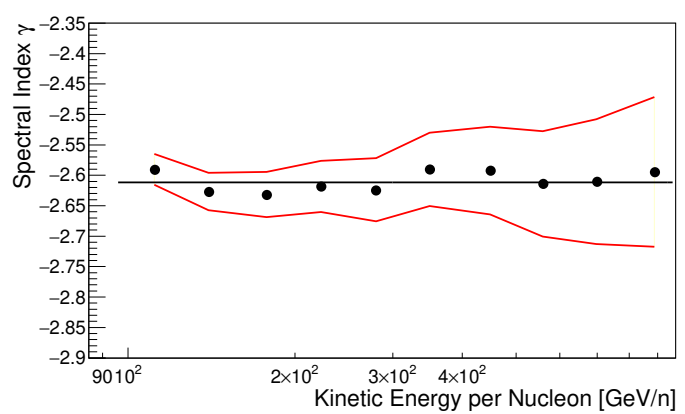

Figure 6: Energy dependence of the spectral index calculated within a sliding energy window for the CALET iron data.The fit with a constant function gives a mean spectral index value $\langle\gamma\rangle=-2.61 \pm 0.01$.

index $\gamma=-2.60 \pm 0.03$ in the energy range between $50 \mathrm{GeV} / n$ and $2 \mathrm{TeV} / n$. Beyond this limit, the uncertainties given by the present statistics and large systematics do not allow to draw a significant conclusion on a possible deviation from a single power law. The resulting data turn out to be consistent with most of the previous measurements within the uncertainty error band, both in spectral shape and normalization.

\section{References}

[1] O. Adriani et al. Phys. Rev. Lett. 119 (2017) 181101.

[2] O. Adriani et al. Phys. Rev. Lett. 120 (2018) 261102.

[3] M. Aguilar et al. Phys. Rev. Lett. 114 (2015) 171103.

[4] M. Aguilar et al. Phys. Rev. Lett. 115 (2015) 211101.

[5] O. Adriani et al. Science 332 (2011) 69.

[6] Y.S. Yoon et al. Astrophys. J. 722 (2011) 122.

[7] Y.S. Yoon et al. Astrophys. J. 839 (2017) 5.

[8] Q. An et al. Sci. Adv. 5 (2019) eaax3793.

[9] O. Adriani et al. Phys. Rev. Lett. 122 (2019) 181102.

[10] M. Aguilar et al. Phys. Rev. Lett. 119 (2017) 251101.

[11] M. Aguilar et al. Phys. Rev. Lett. 120 (2018) 021101.

[12] M. Aguilar et al. Phys. Rev. Lett. 124 (2020) 211102.

[13] H.S. Ahn et al. Astrophys. J. Lett. 714 (2010) L89.

[14] O. Adriani et al. Phys. Rev. Lett. 125 (2020) 251102.

[15] P. Serpico in Proceedings of Science (ICRC2015) 009, 2015. 
[16] N. Tomassetti Astrophys. J. Lett. 752 (2012) L13.

[17] P. Blasi et al. Phys. Rev. Lett. 109 (2012) 061101.

[18] C. Evoli et al. Phys. Rev. D 99 (2019) 103023.

[19] Y. Ohira et al. Phys. Rev. D 93 (2016) 083001.

[20] A. Vladimirov et al. Astrophys. J. 752 (2012) 68.

[21] S. Thoudam and J.R. Hörandel Astron. Astrophys. 567 (2014) A33.

[22] CALET collaboration in Proceedings of Science (ICRC2017) 208, 2017.

[23] Y. Akaike in Proceedings of Science (ICRC2015) 613, 2015.

[24] K. Kasahara in Proc. of 24th ICRC, vol. 1, p. 399, 1995.

[25] See EPICS webpage http://cosmos.n. kanagawa-u.ac.jp/EPICSHome/.

[26] S. Roesler et al. in Proceedings of the Monte Carlo Conference, Lisbon, 1033-1038, 2000.

[27] T.T. Böhlen et al. Nuclear Data Sheets 120 (2014) 211.

[28] Y. Asaoka et al. Astroparticle Physics 91 (2017) 1.

[29] O. Adriani et al. Phys. Rev. Lett. 126 (2021) 241101.

[30] G. D’Agostini Nucl. Instr. and Meth. A 362 (1995) 487.

[31] A. Panov et al. Bull. Russian Acad. Sci. 73 (2009) 564.

[32] M. Ave et al. Astrophys. J. 678 (2008) 262-273.

[33] H.S. Ahn et al. Astrophys. J. 707 (2009) 593.

[34] V. Grebenyuk et al. Adv. in Space Res. 64 (2019) 2546.

[35] M. Ichimura et al. Phys. Rev. D 48 (1993) 1949.

[36] F. Aharonian et al. Phys. Rev. D 75 (2007) 042004.

[37] J.J. Engelmann et al. Astron. Astrophys. 233 (1990) 96.

[38] D. Müller et al. Astrophys. J. 374 (1991) 356.

[39] M. Aguilar et al. Phys. Rev. Lett. 126 (2021) 041104. 


\section{Full Authors List: CALET Collaboration}

O. Adriani ${ }^{1,2}$, Y. Akaike ${ }^{3,4}$, K. Asano ${ }^{5}$, Y. Asaoka ${ }^{5}$, E. Berti ${ }^{1,2}$, G. Bigongiari ${ }^{6,7}$, W. R. Binns ${ }^{8}$, M. Bongi ${ }^{1,2}$, P. Brogi ${ }^{6,7}$, A. Bruno ${ }^{9,10}$, J. H. Buckley ${ }^{8}$, N. Cannady ${ }^{11,12,13}$, G. Castellini ${ }^{14}$, C. Checchia ${ }^{6}$, M. L. Cherry ${ }^{15}$, G. Collazuol ${ }^{16,17}$, K. Ebisawa ${ }^{18}$, A. W. Ficklin ${ }^{15}$, H. Fuke ${ }^{18}$, S. Gonzi ${ }^{1,2}$, T. G. Guzik ${ }^{15}$, T. Hams ${ }^{11}$, K. Hibino ${ }^{19}$, M. Ichimura ${ }^{20}$, K. Ioka ${ }^{21}$, W. Ishizaki ${ }^{5}$, M. H. Israel ${ }^{8}$, K. Kasahara ${ }^{22}$, J. Kataoka ${ }^{23}$, R. Kataoka ${ }^{24}$, Y. Katayose ${ }^{25}$, C. Kato ${ }^{26}$, N. Kawanaka ${ }^{27,28}$, Y. Kawakubo ${ }^{15}$, K. Kobayashi ${ }^{3,4}$, K. Kohri ${ }^{29}$, H. S. Krawczynski ${ }^{8}$, J. F. Krizmanic ${ }^{11,12,13}$, P. Maestro ${ }^{6,7}$, P. S. Marrocchesi ${ }^{6,7}$, A. M. Messineo ${ }^{30,7}$, J.W. Mitchell ${ }^{12}$, S. Miyake ${ }^{32}$, A. A. Moiseev ${ }^{33,12,13}$, M. Mori ${ }^{34}$, N. Mori ${ }^{2}$, H. M. Motz ${ }^{35}$, K. Munakata ${ }^{26}$, S. Nakahira ${ }^{18}$, J. Nishimura ${ }^{18}$, G. A. de Nolfo ${ }^{9}$, S. Okuno ${ }^{19}$, J. F. Ormes ${ }^{36}$ N. Ospina ${ }^{16,17}$, S. Ozawa ${ }^{37}$, L. Pacini 1 14,2 , P. Papini $^{2}$, B. F. Rauch ${ }^{8}$, S. B. Ricciarini ${ }^{14,2}$, K. Sakai ${ }^{11,12,13}$, T. Sakamoto ${ }^{38}$, M. Sasaki ${ }^{33,12,13}$, Y. Shimizu ${ }^{19}$, A. Shiomi ${ }^{39}$, P. Spillantini ${ }^{1}$, F. Stolzi ${ }^{6,7}$, S. Sugita ${ }^{38}$, A. Sulaj 6,7 , M. Takita ${ }^{5}$, T. Tamura ${ }^{19}$, T. Terasawa ${ }^{40}$, S. Torii ${ }^{3}$, Y. Tsunesada ${ }^{41}$, Y. Uchihori $^{42}$, E. Vannuccini ${ }^{2}$, J. P. Wefel ${ }^{15}$, K. Yamaoka $^{43}$, S. Yanagita ${ }^{44}$, A. Yoshida ${ }^{38}$, K. Yoshida ${ }^{22}$, and W. V. Zober ${ }^{8}$

${ }^{1}$ Department of Physics, University of Florence, Via Sansone, 1, 50019 Sesto, Fiorentino, Italy, ${ }^{2}$ INFN Sezione di Florence, Via Sansone, 1, 50019 Sesto, Fiorentino, Italy, ${ }^{3}$ Waseda Research Institute for Science and Engineering, Waseda University, 17 Kikuicho, Shinjuku, Tokyo 162-0044, Japan, ${ }^{4}$ JEM Utilization Center, Human Spaceflight Technology Directorate, Japan Aerospace Exploration Agency, 2-1-1 Sengen, Tsukuba, Ibaraki 305-8505, Japan, ${ }^{5}$ Institute for Cosmic Ray Research, The University of Tokyo, 5-1-5 Kashiwa-no-Ha, Kashiwa, Chiba 277-8582, Japan, ${ }^{6}$ Department of Physical Sciences, Earth and Environment, University of Siena, via Roma 56, 53100 Siena, Italy, ${ }^{7}$ INFN Sezione di Pisa, Polo Fibonacci, Largo B. Pontecorvo, 3, 56127 Pisa, Italy, ${ }^{8}$ Department of Physics and McDonnell Center for the Space Sciences, Washington University, One Brookings Drive, St. Louis, Missouri 63130-4899, USA, ${ }^{9}$ Heliospheric Physics Laboratory, NASA/GSFC, Greenbelt, Maryland 20771, USA, ${ }^{10}$ Department of Physics, Catholic University of America, Washington, DC 20064, USA, ${ }^{11}$ Center for Space Sciences and Technology, University of Maryland, Baltimore County, 1000 Hilltop Circle, Baltimore, Maryland 21250, USA, ${ }^{12}$ Astroparticle Physics Laboratory, NASA/GSFC, Greenbelt, Maryland 20771, USA, ${ }^{13}$ Center for Research and Exploration in Space Sciences and Technology, NASA/GSFC, Greenbelt, Maryland 20771, USA, ${ }^{14}$ Institute of Applied Physics (IFAC), National Research Council (CNR), Via Madonna del Piano, 10, 50019 Sesto, Fiorentino, Italy, ${ }^{15}$ Department of Physics and Astronomy, Louisiana State University, 202 Nicholson Hall, Baton Rouge, Louisiana 70803, USA, ${ }^{16}$ Department of Physics and Astronomy, University of Padova, Via Marzolo, 8, 35131 Padova, Italy, ${ }^{17}$ INFN Sezione di Padova, Via Marzolo, 8, 35131 Padova, Italy, ${ }^{18}$ Institute of Space and Astronautical Science, Japan Aerospace Exploration Agency, 3-1-1 Yoshinodai, Chuo, Sagamihara, Kanagawa 252-5210, Japan, ${ }^{19}$ Kanagawa University, 3-27-1 Rokkakubashi, Kanagawa, Yokohama, Kanagawa 221-8686, Japan, ${ }^{20}$ Faculty of Science and Technology, Graduate School of Science and Technology,, Hirosaki University, 3, Bunkyo, Hirosaki, Aomori 036-8561, Japan, ${ }^{21}$ Yukawa Institute for Theoretical Physics, Kyoto University, Kitashirakawa Oiwakecho, Sakyo, Kyoto 606-8502, Japan, ${ }^{22}$ Department of Electronic Information Systems, Shibaura Institute of Technology, 307 Fukasaku, Minuma, Saitama 337-8570, Japan, ${ }^{23}$ School of Advanced Science and Engineering, Waseda University, 3-4-1 Okubo, Shinjuku, Tokyo 169-8555, Japan, ${ }^{24}$ National Institute of Polar Research, 10-3, Midori-cho, Tachikawa, Tokyo 190-8518, Japan, ${ }^{25}$ Faculty of Engineering, Division of Intelligent Systems Engineering, Yokohama National University, 79-5 Tokiwadai, Hodogaya, Yokohama 240-8501, Japan, ${ }^{26}$ Faculty of Science, Shinshu University, 3-1-1 Asahi, Matsumoto, Nagano 390-8621, Japan, ${ }^{27}$ Hakubi Center, Kyoto University, Yoshida Honmachi, Sakyo-ku, Kyoto 606-8501, Japan, ${ }^{28}$ Department of Astronomy, Graduate School of Science, Kyoto University, Kitashirakawa Oiwake-cho, Sakyo-ku, Kyoto 6068502, Japan, ${ }^{29}$ Institute of Particle and Nuclear Studies, High Energy Accelerator Research Organization, 1-1 Oho, Tsukuba, Ibaraki 305-0801, Japan, ${ }^{30}$ University of Pisa, Polo Fibonacci, Largo B. Pontecorvo, 3, 56127 Pisa, Italy, ${ }^{31}$ Astroparticle Physics Laboratory, NASA/GSFC, Greenbelt, Maryland 20771, USA, ${ }^{32}$ Department of Electrical and Electronic Systems Engineering, National Institute of Technology, Ibaraki College, 866 Nakane, Hitachinaka, Ibaraki 312-8508, Japan ${ }^{33}$ Department of Astronomy, University of Maryland, College Park, Maryland 20742, USA, ${ }^{34}$ Department of Physical Sciences, College of Science and Engineering, Ritsumeikan University, Shiga 525-8577, Japan, ${ }^{35}$ Faculty of Science and Engineering, Global Center for Science and Engineering, Waseda University, 3-4-1 Okubo, Shinjuku, Tokyo 169-8555, Japan, ${ }^{36}$ Department of Physics and Astronomy, University of Denver, Physics Building, Room 211, 2112 East Wesley Avenue, Denver, Colorado 80208-6900, USA, ${ }^{37}$ Quantum ICT Advanced Development Center, National Institute of Information and Communications Technology, 4-2-1 Nukui-Kitamachi, Koganei, Tokyo 184-8795, Japan, ${ }^{38}$ College of Science and Engineering, Department of Physics and Mathematics, Aoyama Gakuin University, 5-10-1 Fuchinobe, Chuo, Sagamihara, Kanagawa 252-5258, Japan, ${ }^{39}$ College of Industrial Technology, Nihon University, 1-2-1 Izumi, Narashino, Chiba 275-8575, Japan ${ }^{40}$ RIKEN, 2-1 Hirosawa, Wako, Saitama 351-0198, Japan, ${ }^{41}$ Division of Mathematics and Physics, Graduate School of Science, Osaka City University, 3-3-138 Sugimoto, Sumiyoshi, Osaka 558-8585, Japan, ${ }^{42}$ National Institutes for Quantum and Radiation Science and Technology, 4-9-1 Anagawa, Inage, Chiba 263-8555, Japan, ${ }^{43}$ Nagoya University, Furo, Chikusa, Nagoya 464-8601, Japan, ${ }^{44}$ College of Science, Ibaraki University, 2-1-1 Bunkyo, Mito, Ibaraki 310-8512, Japan 